\title{
Recent investigations of QCD at HERA
}

\author{
Matthew Wing ${ }^{* \dagger}$ \\ University College London \\ E-mail: m.wing@ucl.ac.uk
}

\begin{abstract}
The latest results from the $\mathrm{H} 1$ and ZEUS collaborations which challenge the QCD description of high energy ep collisions are presented. Data from HERA continue to provide precision measurements and are compared to the latest theoretical predictions. Measurements of new processes are also presented as well as investigation of regions where perturbative QCD fails to describe the data. Four themes are presented here. Measurements of hard QCD processes, prompt photon and jet production, are used to compare to the latest theoretical predictions and, in the case of jet production, used to make high-precision extractions of the strong coupling constant up to next-next-to-leading order in QCD. All H1 and ZEUS charm and beauty cross sections in deep inelastic scattering have been combined and used to extract heavy-quark masses, including the running of the charm-quark mass with the scale of the process. Factorisation in diffraction has been investigated in charm production in deep inelastic scattering and prompt photon production in diffractive photoproduction has been measured for the first time. Finally, the inclusive data on deep inelastic scattering is presented in various forms in order to allow investigation of the underlying mechanism at very low photon virtuality $Q^{2}$ and low Bjorken $x$.
\end{abstract}

XXV International Workshop on Deep-Inelastic Scattering and Related Subjects

3-7 April 2017

University of Birmingham, UK

\footnotetext{
* Speaker.

${ }^{\dagger}$ Also at DESY.
} 


\section{Introduction}

The HERA accelerator is still to date the world's only electron-proton collider and provided high energy collisions recorded by the H1 and ZEUS collaborations. As with all high energy colliders, a wide range of physics has been investigated, with searches for new high energy phenomena, such as supersymmetry, precision measurements of particle properties, and, in particular, a deeper understanding of the strong interaction and its description, embodied in QCD. Given the collision of a point-like object with the hadron, the investigation of the structure of the proton has been central to the HERA programme, with extension in low Bjorken $x$ and high virtualities, $Q^{2}$, by several orders of magnitude compared to fixed-target experiments.

Although HERA finished data taking 10 years ago, H1 and ZEUS have published over 130 papers since then, many investigating various aspects of QCD. Precision measurements continue to be performed which can be used to extract fundamental QCD parameters such as the strong coupling constant, $\alpha_{s}$. New processes continue to be measured for the first time, challenging theoretical calculations. Recent precision and first-time measurements and their understanding in QCD will be discussed in these proceedings.

The nominal parameters for HERA were the collision of protons with energy, $E_{p}$, of $920 \mathrm{GeV}$ and electrons with energy, $E_{e}$, of $27.5 \mathrm{GeV}$, yielding a centre-of-mass energy, $\sqrt{s}$, of $318 \mathrm{GeV}$. The collider ran during the period 1992-2007, with $E_{p}=820$ in the initial years and lower values of $E_{p}$ in 2007 in order to measure the longitudinal structure function, $F_{\mathrm{L}}$. Each of the two experiments, collected about $500 \mathrm{pb}^{-1}$ of data, with some measurements based on a combined sample of $1 \mathrm{fb}^{-1}$. Both H1 and ZEUS had reasonably standard detectors for collider physics, although with more instrumentation in the direction of the proton beam.

\section{Prompt photon production in deep inelastic scattering}

Although the simplest process in which a high energy photon is produced in $e p$ collisions is a purely electromagnetic reaction, the measurement of the prompt photon production cross section is sensitive to QCD through possible higher-order processes. The measurements are also sensitive to the partonic densities in the proton or incoming photon as well as the "Pomeron", the object sometimes used to describe the colourless exchange in diffraction. Prompt photon production within the Standard Model can often be a background to searches for new physics and so its production needs to be understood.

The measurement of the production of prompt photons is complementary to measurements of jet production (see Section 3). Although the samples are much smaller, the study of prompt photons has a number of advantages: an isolated photon produces a clear experimental signature; it is produced in the hard scatter and is not subject to hadronisation; and there are fewer possible diagrams compared to parton-parton scattering.

Prompt photon production with an accompanying hadronic jet in deep inelastic scattering [1] is here presented in comparison to various models. In Fig. 1, cross sections are shown as a function of the fraction of the incoming photon's momentum that takes part in the production of the photon and jet, $x_{\gamma}$, the fraction of the proton's momentum that takes part in the production of the photon and jet, $x_{p}$, the pseudorapidity difference between the jet and photon, $\Delta \eta$, and the azimuthal 
difference between the scattered electron and photon, $\Delta \phi_{e, \gamma}$. The data are compared with two theoretical predictions: a next-to-leading order (NLO) QCD calculation by Aurenche, Fontannaz and Guillet [2], based on collinear factorisation; and a calculation by Baranov, Lipatov and Zotov [3], based on $k_{T}$ factorisation. The prediction from BLZ does not describe the data in $x_{\gamma}$ and $\Delta \eta$, but gives a good description of the other variables (including others not shown). The AFG prediction describes the data reasonably well. When normalised to the data, the PythiA [4] Monte Carlo simulation gives a good description (not shown here) of the data.
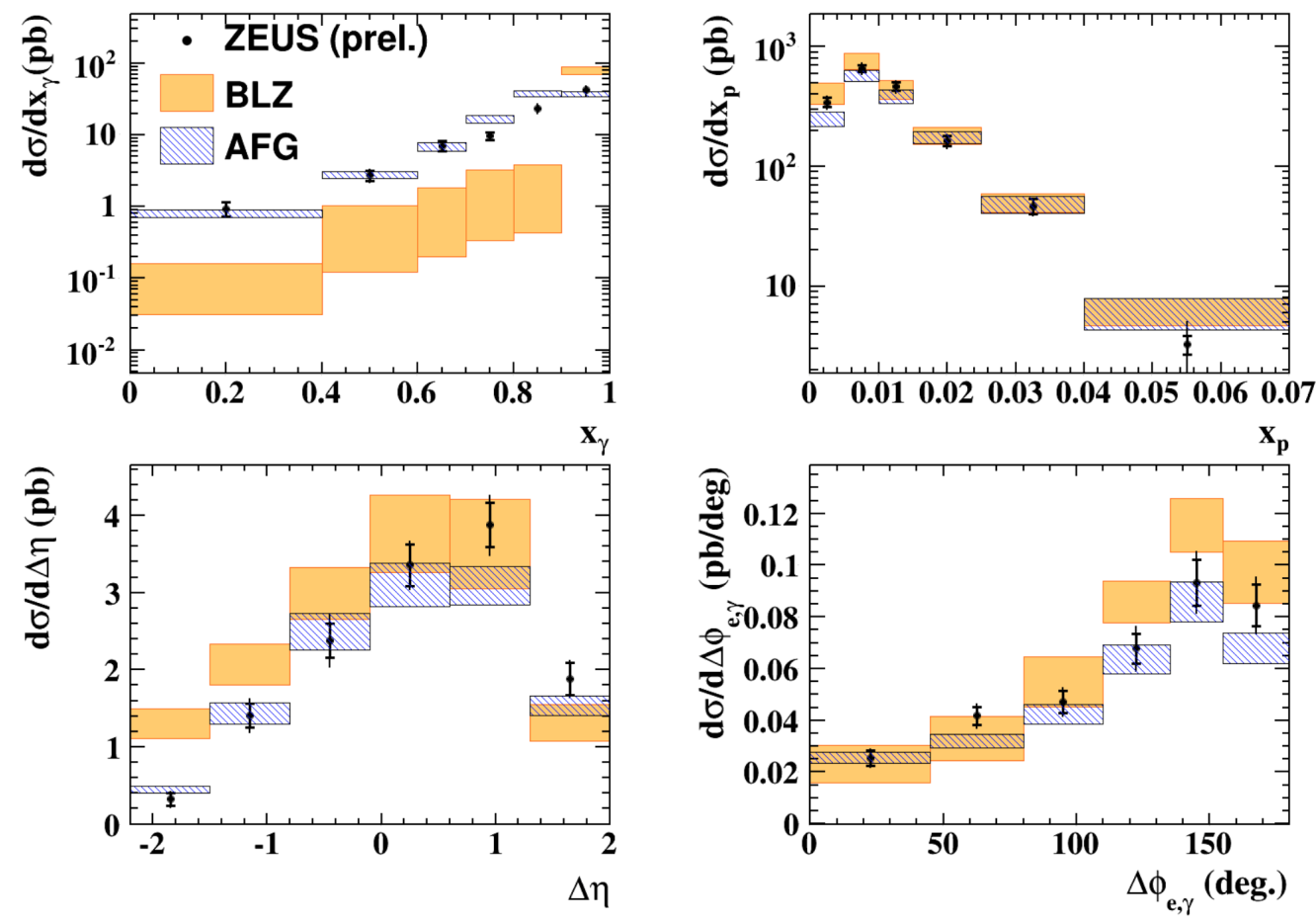

Figure 1: Differential cross sections for various variables in prompt photon production in deep inelastic scattering. The data are compared to theoretical predictions from Aurenche et al. (AFG) and from Baranov et al. (BLZ), both of which are corrected for hadronisation using the PYTHIA Monte Carlo model. Plot taken from [1].

\section{Jet production in deep inelastic scattering}

Jet production in deep inelastic scattering at HERA provides one of the most powerful tests of QCD, with sensitivity to the PDFs and precise extraction of the strong coupling constant, $\alpha_{s}$, possible. The $\mathrm{H} 1$ collaboration have recently published such a measurement $[5,6]$ using about $300 \mathrm{pb}^{-1}$ and compared the data to $\mathrm{PQCD}$ calculations up to next-to-next-to-leading order (NNLO) [7]. The measurement was performed over a wide kinematic range, $5.5<Q^{2}<15000 \mathrm{GeV}^{2}$; in Fig. 2 cross sections are shown in comparison to different theoretical predictions. The NLO QCD predictions describe the data reasonably well, although the theoretical uncertainties are relatively large, particularly at low $Q^{2}$. The NNLO QCD predictions give an improved description and have smaller uncertainties due to the variation in the scales. 

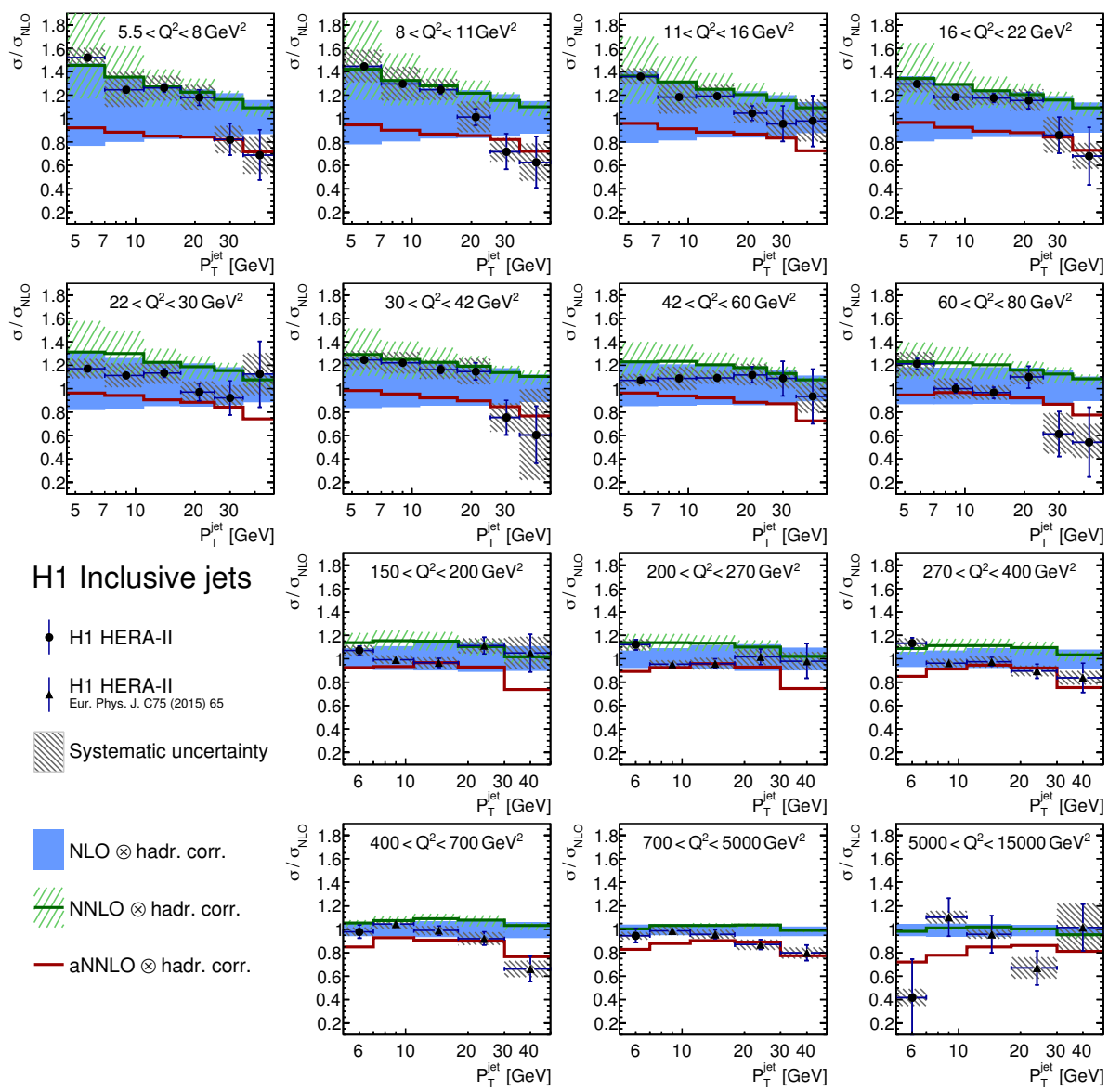

Figure 2: Ratio of inclusive-jet cross sections to NLO QCD predictions and ratio of aNNLO and NNLO QCD to NLO QCD predictions as a function of $Q^{2}$ and jet transverse momentum, $P_{T}^{\text {jet }}$. Plot taken from [5].

The data and theory have been used to extract values of $\alpha_{s}$ at NLO and NNLO. The extractions agree with other extractions and with the world average, although the value using NNLO QCD is a little lower at $\alpha_{s}\left(M_{Z}\right)=0.1157(6)_{\exp }\left({ }_{-26}^{+31}\right)_{\text {theo. }}$. As can be seen from the numbers, the experimental uncertainty is about $0.5 \%$, whereas the theoretical uncertainty, even at NNLO, is significantly large at about $2.5 \%$, although reduced from about $4 \%$ at NLO.

\section{Extraction of the heavy-quark masses}

Heavy-quark production at HERA is dominated by boson-gluon fusion and as such is directly sensitive to the gluon density in the proton. Given the masses of the heavy quarks, perturbative QCD predictions are expected to be reliable, although along with $Q^{2}$ and the transverse momentum of the heavy quarks, there are many scales in the process which poses an extra challenge to QCD calculations.

Heavy-quark production in deep inelastic scattering is included in QCD fits to the parton densities in the proton and these analyses provide strong constraints on the masses of the heavy quarks. Indeed the data can be used to extract the mass of the charm quark at several scales and investigate 
its evolution or "running" with scale [8], as is done for $\alpha_{s}$. The mass as a function of the energy scale is shown in Fig. 3, where the running of the charm-quark mass is clearly observed.

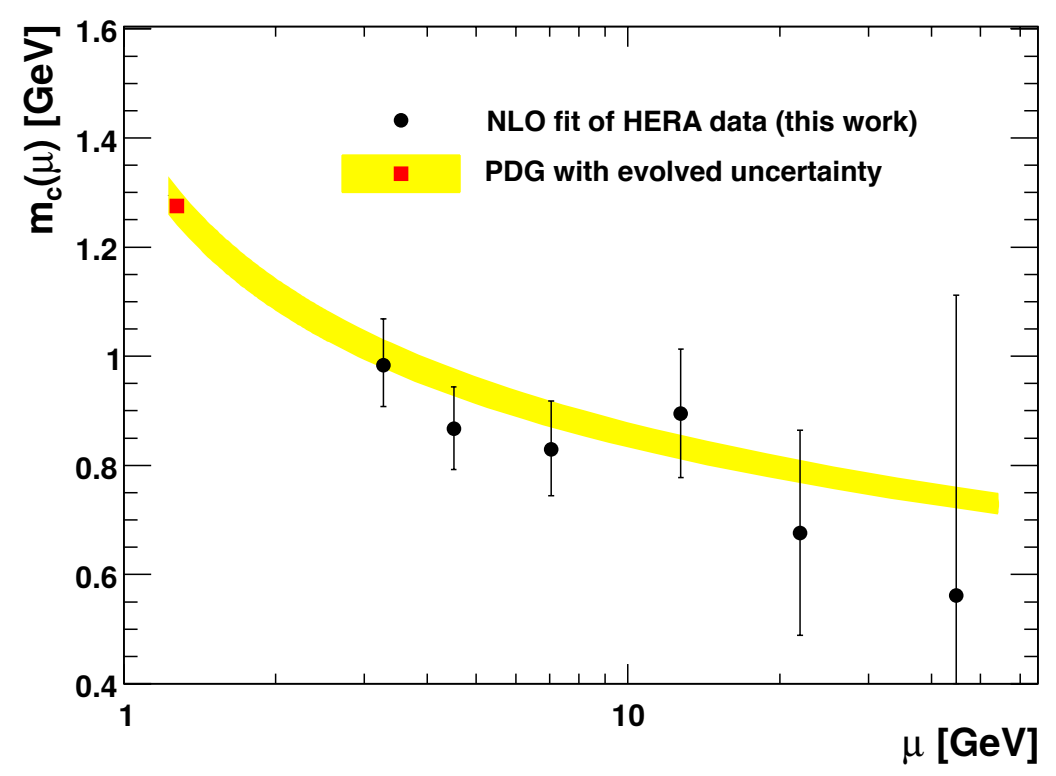

Figure 3: Charm-quark mass, $m_{c}(\mu)$, as a function of the scale extracted from HERA data and compared to the PDG world average and its running evolved to higher scales. Plot taken from [8].

All of the charm data in deep inelastic scattering from H1 and ZEUS has been combined to form a common HERA data set [9], including three new high-precision sets compared to the previous combination [10]. The beauty data in deep inelastic scattering has been combined for the first time [9]. Correlations between all data sets have been accounted for. The data sets are consistent $\left(\chi^{2} /\right.$ dof $\left.=149 / 187\right)$ and the combination leads to an improved precision, as can be seen in Fig. 4.

The data are compared with NLO QCD predictions using different PDFs in Fig. 5. The description of the data is reasonable, although some difference in shape is seen, in particular for $Q^{2}=12 \mathrm{GeV}^{2}$. The NLO QCD analysis of the data ("NLO fit DIS $+\mathrm{c}+\mathrm{b}$ " in Fig. 5) yielded masses of the heavy quarks:

$$
\begin{aligned}
& m_{c}\left(m_{c}\right)=1290_{-41}^{+46}(\text { fit })_{-14}^{+62}(\text { model })_{-31}^{+7}(\text { param }) \mathrm{MeV} \\
& m_{b}\left(m_{b}\right)=4049_{-109}^{+104}(\text { fit })_{-32}^{+90}(\text { model })_{-31}^{+1}(\text { param }) \mathrm{MeV}
\end{aligned}
$$

The results compare well with the PDG values $\left(m_{c}\left(m_{c}\right)=1280 \pm 30 \mathrm{MeV}\right.$ and $m_{b}\left(m_{b}\right)=$ $\left.4180_{-30}^{+40} \mathrm{MeV}\right)[14]$ and the charm mass in particular has competitive uncertainties. The uncertainty on the extracted charm-quark mass presented here is dominated by variation of the scale in the QCD calculation. It is hoped that improved, or higher-order, calculations will have an impact on the measurements, similar to that seen in the jet results (see Section 3), and lead to an even more competitive extraction of the charm-quark mass. 


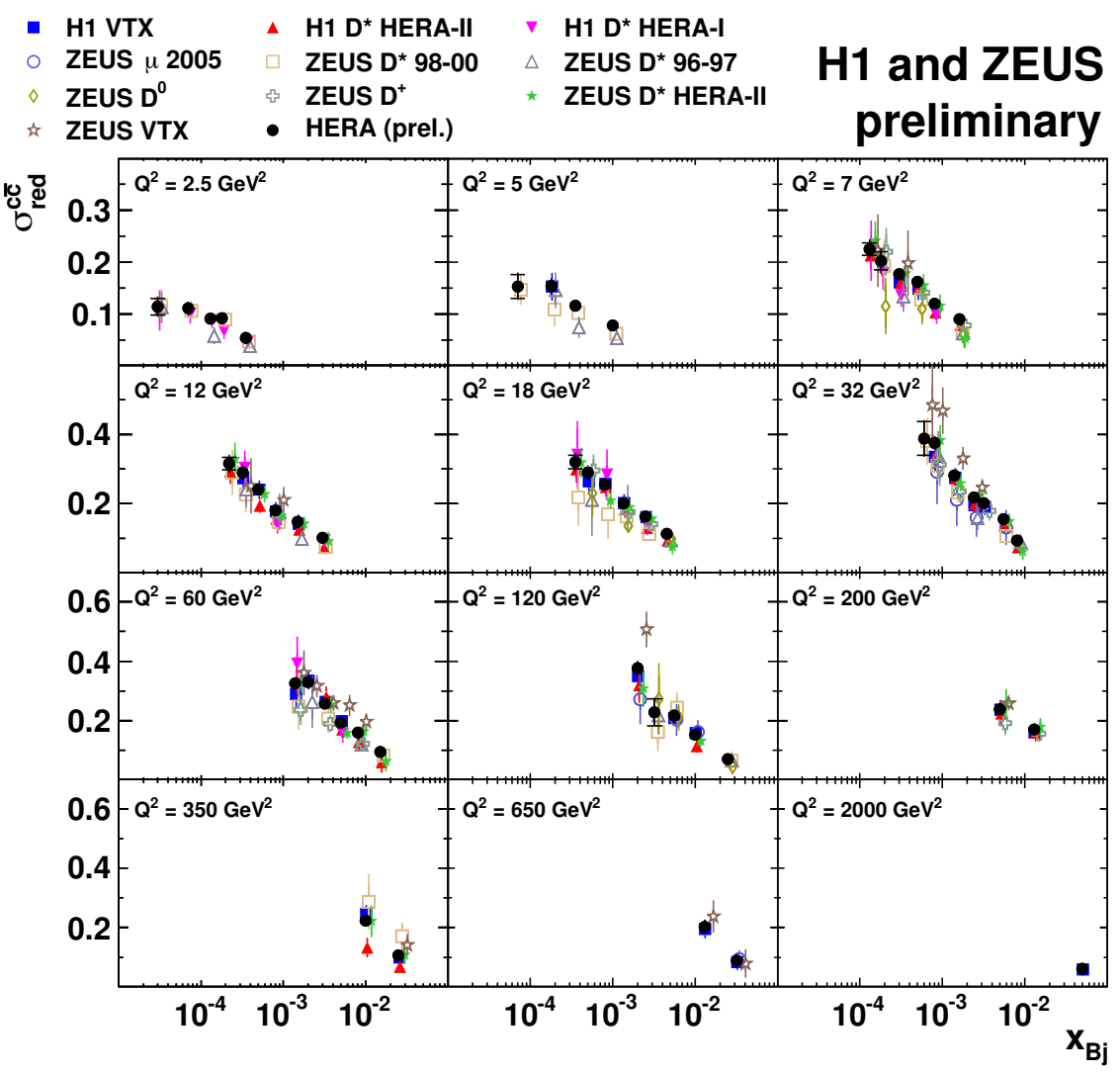

Figure 4: Individual and combined cross sections for charm production in deep inelastic scattering. The cross sections are shown as a function of Bjorken $x$ at fixed $Q^{2}$. Plot taken from [9].

\section{Diffraction and factorisation}

The observation and subsequent detailed investigation of diffraction in high energy ep collisions is one of the highlights of the HERA programme. The measurements from HERA reinvigorated this area of physics and still presents theory with a number of challenges such as the interplay between hard and soft physics, the question of factorisation and, in general, the nature of the underlying process. The expectation of factorisation in diffractive deep inelastic scattering [15] means that diffraction is amenable to QCD in which a hard sub-process is convoluted with the PDFs of the diffractive exchange, or "Pomeron", following a similar approach to that used for the proton in inclusive deep inelastic scattering.

As a result of factorisation, the diffractive PDFs extracted by fitting inclusive diffraction data should be applicable in other processes, such as when requiring a specific final state or having a different initial collision, e.g. pp. In order to test this, the $\mathrm{H} 1$ collaboration have measured charm production, through reconstructing decays of $D^{*}$ mesons, in diffractive deep inelastic scattering using a luminosity of about $300 \mathrm{pb}^{-1}$ [16]. The measurements are compared to predictions from NLO QCD with parton densities in the Pomeron extracted from fits [17] to inclusive diffraction 


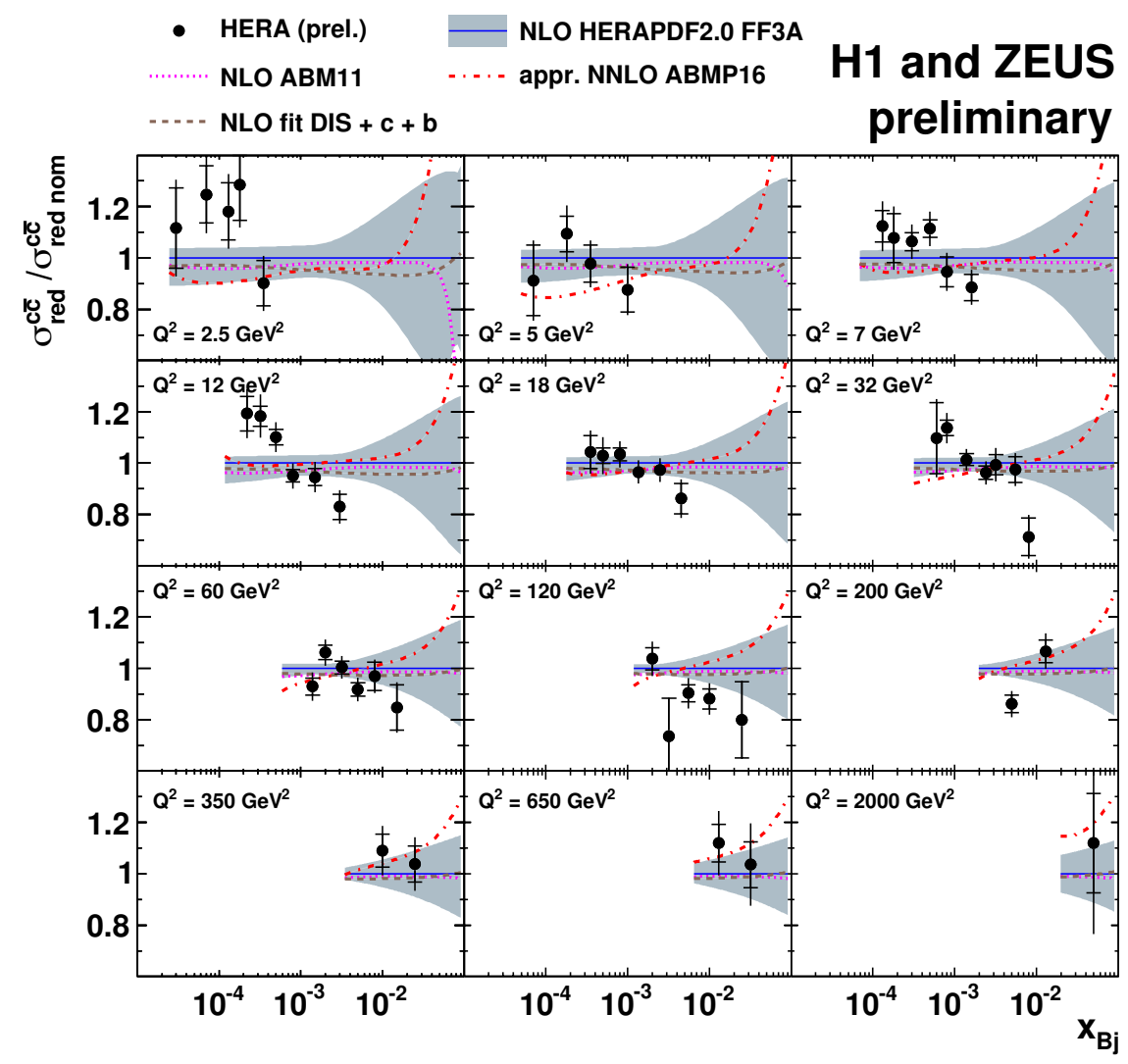

Figure 5: Ratio of measured cross sections for charm production in deep inelastic scattering compared to QCD predictions with different proton PDFs: ABM11 [11], HERAPDF2.0 FF3A [12] and the fit discussed here all at NLO, as well as ABMP16 [13] at approximate NNLO. The cross sections are shown as a function of Bjorken $x$ at fixed $Q^{2}$. The grey bands represent the theory uncertainties. Plot taken from [9].

data in Fig. 6. The variables, characterising the $D^{*}$ meson, viz. its transverse momentum, $p_{t, D^{*}}$, and pseudorapidity, $\eta_{D^{*}}$, are shown to be well described by QCD (as are other kinematic variables, not shown). The conclusions are consistent with other results at HERA in which processes in diffractive deep inelastic scattering are well described by NLO QCD.

In photoproduction, in which the exchanged photon is quasi-real $\left(\left\langle Q^{2}\right\rangle \approx 10^{-4} \mathrm{GeV}^{2}\right)$, the holding of factorisation is not so clear. Results from H1 indicate that NLO QCD cannot describe diffractive jet photoproduction, being roughly a factor of 2 too high, whereas the measurements from ZEUS indicate no such discrepancy [18]. Therefore, it is imperative to measure other processes in photoproduction. A first measurement [19] of diffractive prompt photon photoproduction has been made by ZEUS using a luminosity of $374 \mathrm{pb}^{-1}$ and is shown compared to RAPGAP [20] Monte Carlo expectations in Fig. 7. Of particular note is the distribution of the fraction of the Pomeron energy that takes part in the production of a prompt photon and a jet, $z_{\mathbb{P}}^{\text {meas }}$, which is poorly described by the simulation. The events at high $z_{\mathbb{P}}^{\text {meas }}$ are concentrated at high $x_{\gamma}$ and so are evidence for processes containing a direct photon and direct Pomeron, which is not present in 

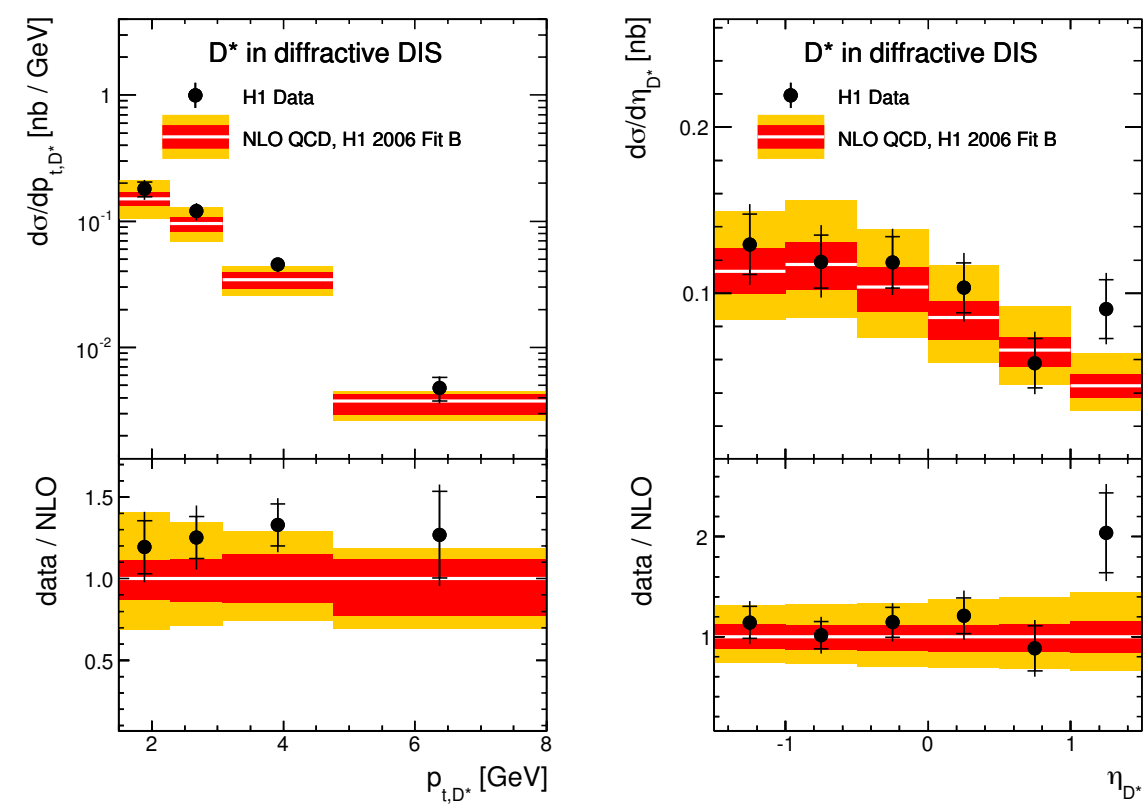

Figure 6: Cross sections for $D^{*}$ variables in diffractive deep inelastic scattering compared to predictions from NLO QCD. The ratio of the data to NLO QCD predictions are also shown. Plot taken from [16].

RAPGAP. Unfortunately, at the time of writing an NLO QCD prediction for this process is not available; it is hoped that a future calculation may shed more light on the situation of factorisation in diffractive photoproduction.

\section{Investigation of low- $Q^{2}$ data}

The recently published final combined HERA data in inclusive deep inelastic scattering [12] provide a wealth of input to understanding QCD, such as being the pre-eminent data set in the extraction of proton PDFs and a precise extraction of $\alpha_{s}$. The data can also be used to understand a region where $\mathrm{pQCD}$ is not expected to describe the data, at low $Q^{2}$ and low Bjorken $x$ [21]. Data at the lowest $Q^{2}$ are shown in Fig. 8, in which the virtual-photon-proton cross section is shown as a function of $Q^{2}$ for fixed photon-proton centre-of-mass energy, $W$. The data show a smooth transition from the higher- $Q^{2}$ (perturbative) to the lower- $Q^{2}$ (non-perturbative) region. These data cannot be described by pQCD predictions at NLO or NNLO, nor can they be described by a Regge model with one pole ("ZEUSREGGE" [22] and the updated "HHT-REGGE" [21]). The model HHT-ALLM [21, 23], which is also based on Regge phenomenology, but with many more parameters, describes the data reasonably well in the full kinematic region.

The cross-section data can also be converted to the structure function, $F_{2}$, although this is a model-dependent procedure and was done with pQCD above a few $\mathrm{GeV}^{2}$ and Regge phenomenology below. The values of $F_{2}$ can then be parametrised as $F_{2}=C\left(Q^{2}\right) x_{\mathrm{Bj}}^{-\lambda\left(Q^{2}\right)}$, where $C\left(Q^{2}\right)$ and $\lambda\left(Q^{2}\right)$ are parameters to be fit for each $Q^{2}$. The extracted values of $\lambda\left(Q^{2}\right)$ are shown in Fig. 9, compared to various expectations. The low- $Q^{2}$ region can be described by simple Regge theory, whereas the high- $Q^{2}$ region can be described by pQCD and the re-fitted HHT-ALLM model de- 


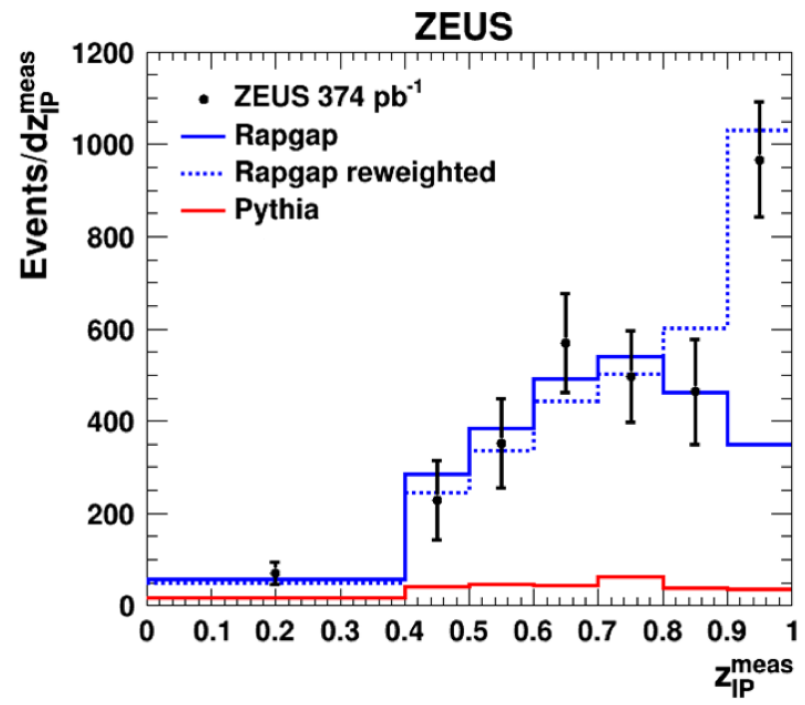

Figure 7: Distribution of $z_{\mathbb{P}}^{\text {meas }}$ in ZEUS diffractive prompt photon photoproduction data compared to Monte Carlo simulations. The default RAPGAP prediction is shown, as well as the prediction re-weighted to fit the $z_{\mathbb{P}}^{\text {meas }}$ distribution. The prediction from PYTHIA shows the non-diffractive background contribution. Plot taken from [19].

scribes the full- $Q^{2}$ region. The overall trend with $Q^{2}$ is in fact rather simple and is well described by a quadratic fit. These and other distributions will prove valuable in further theoretical development and understanding of the physics in this low- $Q^{2}$ and low- $x$ region. The construction of a new ep or $e A$ collider [25] would provide more data to widen the kinematic range of such future studies.

\section{Summary}

Results from HERA continue to challenge our understanding of QCD and, in these proceedings, some recent highlights have been reviewed. The data are compared to the latest predictions, up to next-to-next-to-leading order in QCD and this precision comparison allows world-competitive extractions of fundamental parameters of the strong force such as the strong coupling constant and masses of the heavy quarks. Diffraction poses a special challenge although the validity of factorisation and use of diffractive parton densities is successful for deep inelastic scattering. However, a clear model to describe diffractive physics in the different initial states, deep inelastic scattering and photoproduction, and final states is still being sought. Although perturbative QCD can describe many of the HERA measurements, in particular inclusive deep inelastic scattering, the region of low Bjorken $x$ is still poorly understood and further phenomenological development should be compared to the HERA data summarised here.

\section{Acknowledgements}

The continuing efforts of the HERA collaborations and publication of high-quality results, 10 years after the end of data taking, is truly remarkable. These proceedings would not have been 


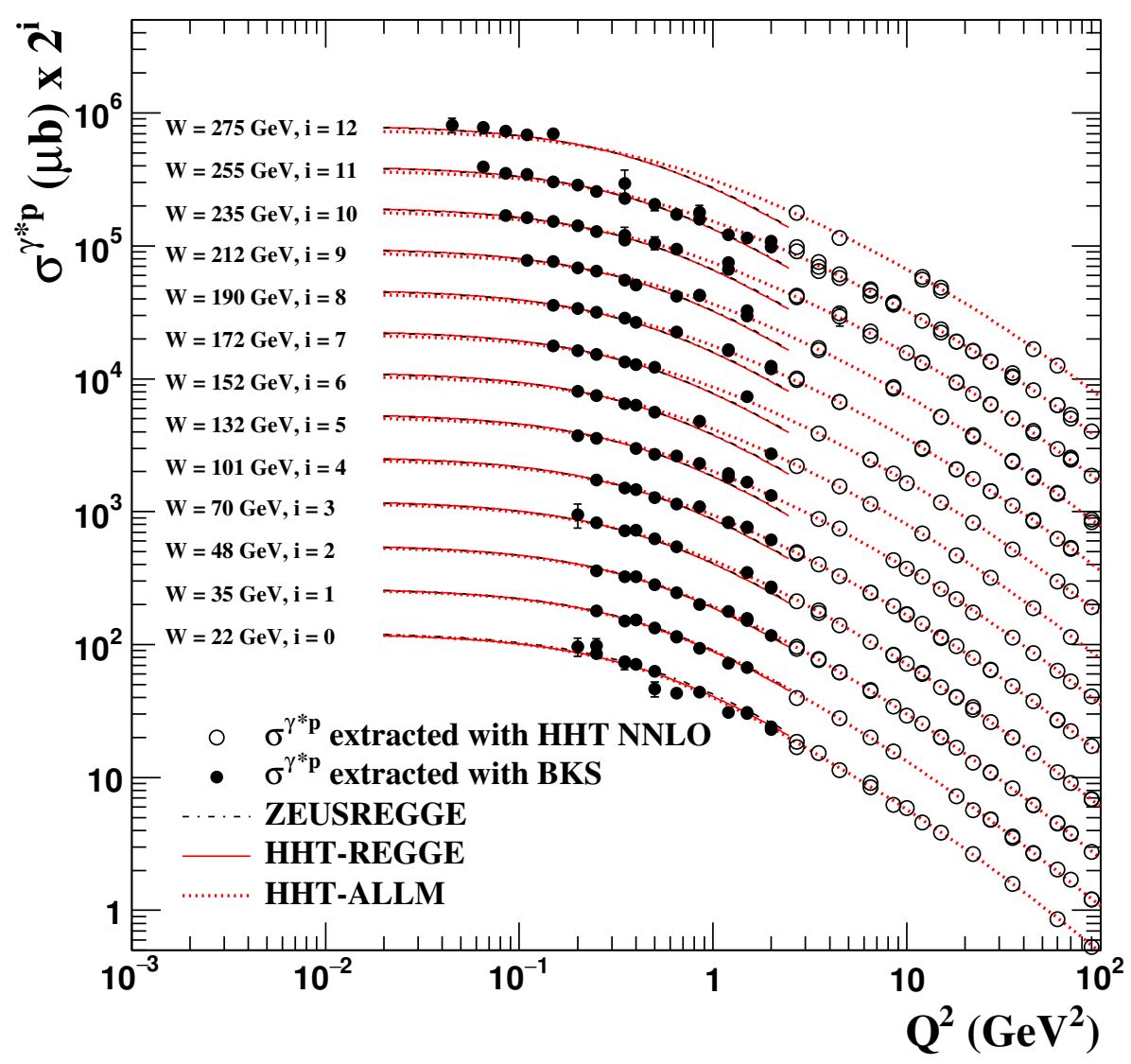

Figure 8: Cross section, $\sigma^{\gamma^{*} p}$, versus $Q^{2}$ at fixed $W$, extracted with BKS at low $Q^{2}$ and with HHT NNLO QCD [24] at high $Q^{2}$. The data are compared with a previous ZEUSREGGE fit as well as a new version, HHT-REGGE, and a new fit based on the ALLM model, HHT-ALLM. Plot taken from [21].

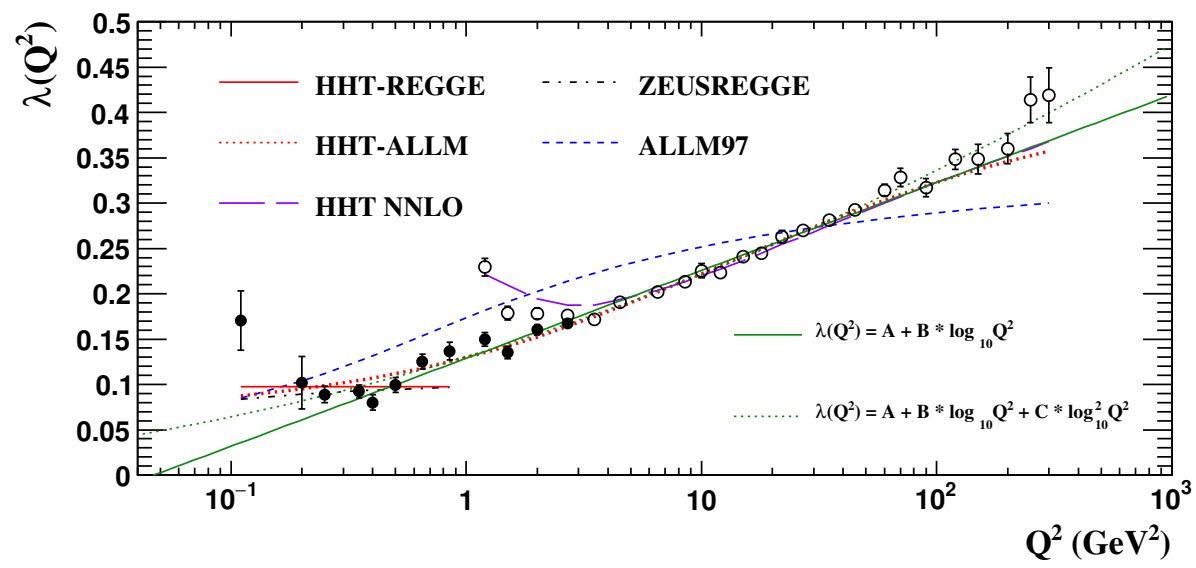

Figure 9: Exponent $\lambda\left(Q^{2}\right)$ versus $Q^{2}$, from fits to $F_{2}$, extracted with NNLO QCD for $Q^{2} \geq 1.2 \mathrm{GeV}^{2}$ (open points) and extracted with a Regge model for $Q^{2} \leq 2.7 \mathrm{GeV}^{2}$ (solid points). The data are compared to various theoretical predictions, as well as a first- and second-order polynomial in $\log Q^{2}$. Plot taken from [21]. 
possible without this and as such are dedicated to the members of $\mathrm{H} 1$ and ZEUS who continue to produce results will challenge our understanding of QCD. The support and hospitality from DESY, Hamburg is gratefully acknowledged as well as support from the Alexander von Humboldt Stiftung.

\section{References}

[1] ZEUS Coll., O. Hlushchenko, these proceedings.

[2] P. Aurenche, M. Fontannaz and J.Ph. Guillet, Eur. Phys. J. C 44 (2005) 395; P. Aurenche and M. Fontannaz, Eur. Phys. J. C 77 (2017) 324.

[3] S. Baranov, A. Lipatov and N. Zotov, Phys. Rev. D 81 (2010) 094034.

[4] T. Sjöstrand et al., JHEP 0605 (2006) 026.

[5] H1 Coll., Eur. Phys. J. C 77 (2017) 215;

[6] H1 Coll. et al., H1prelim-17-031; D. Britzger, these proceedings.

[7] J. Currie, T. Gehrmann and J. Niehues, Phys. Rev. Lett. 117 (2016) 042001; J. Currie et al., JHEP 1707 (2017) 018.

[8] A. Gizhko et al., DESY-17-048, arXiv:1705.08863; A. Geiser, these proceedings.

[9] H1 and ZEUS Coll., H1prelim-17-071, ZEUS-prel-17-01; O. Zenaiev, these proceedings.

[10] H1 and ZEUS Colls., Eur. Phys. J. C 73 (2013) 2311.

[11] S. Alekhin, J. Blümlein and S. Moch, Phys. Rev. D 86 (2012) 054009.

[12] H1 and ZEUS Colls., Eur. Phys. J. C 75 (2015) 580.

[13] S. Alekhin et al., arXiv:1701.05838.

[14] Particle Data Group, C. Patrignani et al., Chin. Phys. C 40 (2016) 100001 and 2017 update.

[15] J.C. Collins, Phys. Rev. D 57 (1998) 3051; erratum, ibid. D 61 (2000) 019902.

[16] H1 Coll., V. Andreev et al., Eur. Phys. J. C 77 (2017) 340; K. Cerny, these proceedings.

[17] H1 Coll., A. Aktas et al., Eur. Phys. J. C 48 (2006) 715.

[18] For the latest results, see: H1 Coll., JHEP 1505 (2015) 056; ZEUS Coll., Eur. Phys. J. C 55 (2008) 177.

[19] ZEUS Coll., H. Abramowicz et al., Phys. Rev. D 96 (2017) 032006; P. Bussey, these proceedings.

[20] H. Jung, https://rapgap.hepforge.org/rapgap.pdf

[21] I. Abt et al., Phys. Rev. D 96 (2017) 014001; K. Wichmann, these proceedings.

[22] ZEUS Coll., J. Breitweg et al., Eur. Phys. J. C 7 (1999) 609.

[23] H. Abramowicz et al., Phys. Lett. B 269 (1991) 465; H. Abramowicz and A. Levy, hep-ph/9712415.

[24] I. Abt et al., Phys. Rev. D 94 (2017) 034032.

[25] A. Caldwell and M. Wing, Eur. Phys. J. C 76 (2016) 463; A. Accardi et al., Eur. Phys. J. A 52 (2016) 268. 